\section{Síndrome hemolítico-urémico asociado al uso de gemcitabina. Caso clínico}

\author{
JORGE VEGA ${ }^{1,2,3}$, CHRISTIAN PARODI $^{4}$, \\ GONZALO P. MÉNDEZ ${ }^{5}$, HELMUTH GOECKE ${ }^{2,3}$
}

\section{Hemolytic-uremic syndrome associated with gemcitabine use. Report of one case}

Gemcitabine is a widely used drug in the treatment of advanced pancreatic cancer and other malignancies. It is generally well tolerated and exceptionally its use has been associated with hemolytic-uremic syndrome, causing acute kidney injury, hypertension, chronic renal failure requiring dialysis, and death. We report a 60-year-old man with pancreatic carcinoma and regional lymph node invasion, whom after four months of therapy with gemcitabine and after dose number 11, suddenly developed an acute nephritic syndrome with moderate renal impairment, associated with severe anemia (hemoglobin $6.0 \mathrm{~g} / \mathrm{dL}$ ) and thrombocytopenia $\left(20,000 \mathrm{~mm}^{3}\right.$ ). Renal biopsy showed the classic findings of thrombotic microangiopathy. Gemcitabine was discontinued and renal function and hematological parameters gradually improved.

(Rev Med Chile 2013; 141: 797-802).

Key words: Acute kidney injury; Anemia; Antimetabolites, antineoplastic; Gemcitabine; Hemolytic-uremic syndrome; Pancreatic neoplasms.

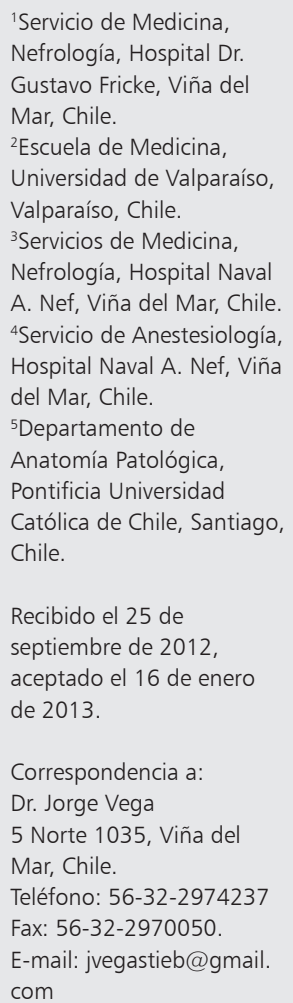

E 1 síndrome hemolítico-urémico (SHU) es una entidad clínica caracterizada por anemia hemolítica microangiopática, trombocitopenia e injuria renal aguda ${ }^{1,2}$. Existen diversas etiologías: infecciones entéricas, formas familiares, enfermedades autoinmunes, trasplante de médula ósea, embarazo, neoplasias diseminadas, infecciones por VIH y drogas ${ }^{3-10}$. De estas últimas se han descrito casos asociados al uso de ciclosporina, tacrolimus, ticlopidina, alfa interferón, quinina y a drogas oncológicas como mitomicina C, cisplatino, bleomicina, 5 fluoracilo, adriamicina, carboplatino y otras $\mathrm{s}^{3-6,10}$.

En los últimos años se han comunicado en forma creciente casos de SHU asociados al uso de gemcitabina (GEM), droga introducida en 1996 para el tratamiento del cáncer pancreático irresecable o metastásico y que ha tenido un amplio uso ${ }^{3-11,16-24}$. Se ha utilizado también en cáncer pulmonar de células no pequeñas, linfoma, cáncer de ovario, mama, hígado, vía biliar y vejiga ${ }^{3-5,14,19}$.

El SHU asociado a GEM tiene una baja inci- dencia, varía entre $0,008 \%$ y 2,2\% de los pacientes tratados con la droga ${ }^{3,10-13,22}$. Se asocia frecuentemente a falla renal con necesidad de diálisis y tiene elevada mortalidad ${ }^{11,14,21,22}$. La búsqueda rutinaria de las manifestaciones clínicas de este síndrome antes de cada administración de una dosis de GEM permite sospechar oportunamente el trastorno y muchas veces revertirlo al suspender su administración, reduciendo la morbilidad y mortalidad ${ }^{17}$.

Comunicamos un caso de SHU por GEM en que el diagnóstico clínico-patológico oportuno permitió obtener una buena evolución, evitando la progresión del daño renal.

\section{Caso clínico}

Paciente de 60 años, sin antecedentes mórbidos, a quien en una tomografía computada de abdomen efectuada por dolor abdominal, se le encontró un carcinoma de $2 \mathrm{~cm}$ localizado en la cabeza del páncreas. Fue sometido a una operación 
de Whipple. Había 2 adenopatías regionales con metástasis de carcinoma y no existía diseminación a distancia. Tres meses después de la operación se inició quimioterapia con GEM. Después de recibir 11 dosis de GEM, transcurridos 4 meses de su inicio y habiendo recibido una dosis total acumulada de $16.400 \mathrm{mg}\left(9.318 \mathrm{mg} / \mathrm{m}^{2}\right)$, presentó edema palpebral y de tobillos e hipertensión arterial de hasta 160/110 mmHg. Los exámenes de laboratorio mostraron hematuria microscópica (26-50 hematíes por campo), proteinuria $(1.740 \mathrm{mg} / 24$ $\mathrm{h})$, cilindruria granulosa, ascenso de la creatinina sérica hasta un máximo de $2,0 \mathrm{mg} / \mathrm{dL}$ con caída del clearance de creatinina a $52 \mathrm{ml} / \mathrm{min} / 1,73 \mathrm{~m}^{2}$ (medido) a los 21 días de la última dosis. Previo a ello se había observado anemia progresiva (hemoglobina $6.0 \mathrm{~g} / \mathrm{dL})$ y trombocitopenia $\left(20.000 \mathrm{~mm}^{3}\right)$, que se atribuyeron a mielodepresión por GEM. La bilirrubinemia total ascendió a $1,79 \mathrm{mg} / \mathrm{dL}$, la directa a $0,93 \mathrm{mg} / \mathrm{dL}$ y la LDH a $701 \mathrm{U} / \mathrm{L}$ (VN: 125-243), lo que se interpretó como secundario a toxicidad hepática por GEM. Se suspendió la administración de nuevas dosis de GEM, se prescribió furosemide para controlar el edema y olmesartán para el manejo de la hipertensión arterial.

Estando el recuento de plaquetas sobre 100.000 $\mathrm{mm}^{3}$ se efectuó una biopsia renal percutánea que mostró hallazgos característicos de una microangiopatía trombótica crónica activa. El examen de microscopia óptica reveló la presencia de glomérulos con numerosos dobles contornos en sus asas capilares y arteriolas, con intensa disminución de su lumen, hiperplasia concéntrica de miocélulas y depósito hialino mural sugerente de material microtrombótico incorporado en su endotelio y pared. El examen de inmunofluorescencia no mostró presencia de complejos inmunes en los glomérulos. La microscopia electrónica confirmó las alteraciones glomerulares encontradas en la microscopia óptica; en especial, se demostró la presencia de múltiples dobles contornos con interposición celular y expansión acentuada de la lámina rara interna de la membrana basal por material electrón-lúcido y detritus subendotelial. Todas estas alteraciones se encontraron en ausencia de depósitos densos de tipo complejo inmune (Figuras 1, 2, 3, 4). La lesión pedicelar fue moderada.

La suspensión de la GEM se acompañó de una mejoría en la función renal y de los parámetros hematológicos. En el último control, 10 sem des- pués de la última dosis de GEM, la hemoglobina fue $12,3 \mathrm{~g} / \mathrm{dL}$, plaquetas $147.000 \mathrm{~mm}^{3}$, leucocitos $4.700 \mathrm{~mm}^{3}$, creatinina sérica $1,18 \mathrm{mg} / \mathrm{dl}$, clearance de creatinina $85 \mathrm{ml} / \mathrm{min} / 1,73 \mathrm{~m}^{2}$ y LDH normal. El paciente continuó recibiendo olmesartán como terapia antihipertensiva originada por el daño renal por GEM.

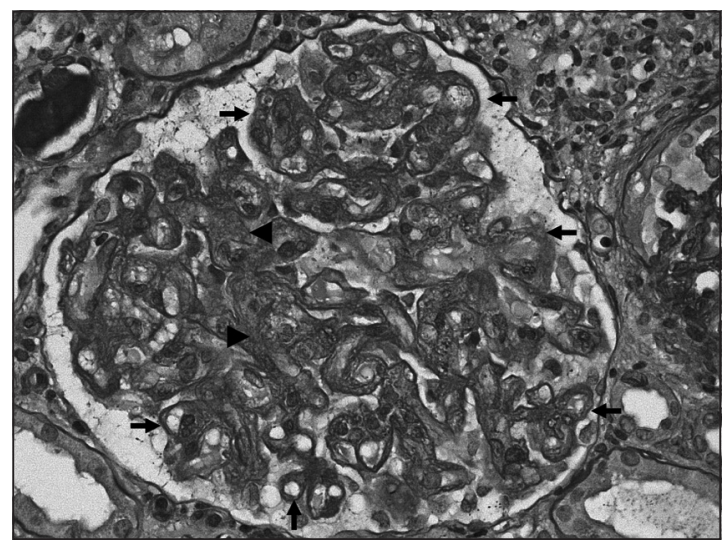

Figura 1. Microscopia óptica de un glomérulo con alteración intensa de su arquitectura mesangiocapilar. Se reconocen numerosos dobles contornos y defectos delaminativos en sus asas capilares libres (flechas). Los ejes mesangiales presentan una matriz PAS positiva débil de aspecto fibrilar (cabezas de flecha). (tinción de PAS, x400).

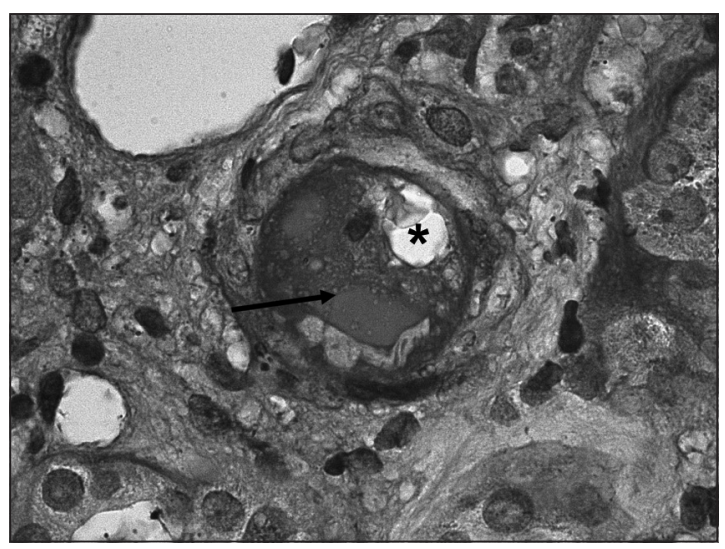

Figura 2. Imagen vista con aceite de inmersión que muestra una arteríola con material hialino (flecha) en su íntima y reemplazando parte de la media, compatible con material trombótico incorporado en su pared. Se observa un lumen residual muy estenótico (asterisco). (tinción de PAS, x600). 


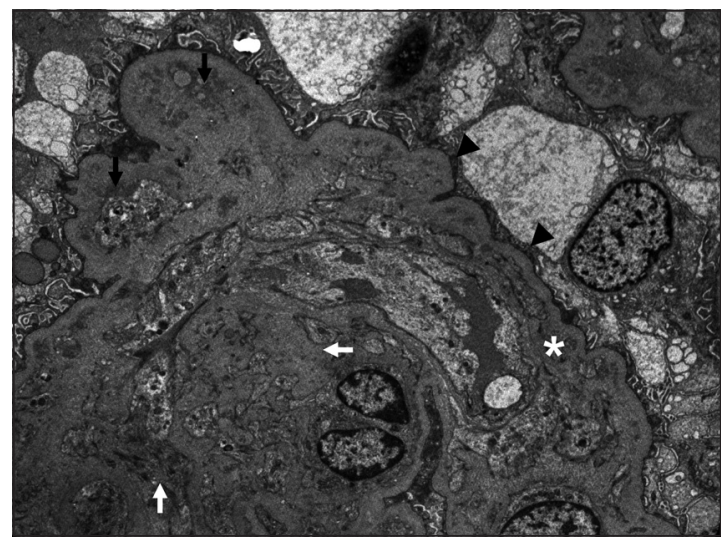

Figura 3. Microscopia electrónica de transmisión. Segmento del ovillo glomerular con remodelación intensa de la membrana basal del asa capilar libre, la cual presenta reduplicación de su membrana basal (asterisco) y acumulación de material denso amorfo (detritus) en la zona subendotelial remanente (flechas negras). El borramiento pedicelar es moderado (cabezas de flecha). El mesangio esboza nodularidad temprana por aumento del material de matriz, detritus y fibrillas colágenas (flechas blancas), compatible con esclerosis de tipo cicatrizal (Tetróxido de osmio-acetato de uranilo, x6.000).

\section{Discusión}

La GEM es un análogo de nucleósidos pirimidínicos que tiene un amplio espectro antitumoral $^{4,6,23}$. Inhibe la síntesis celular de ADN y origina apoptosis en las células tumorales ${ }^{16}$. Habitualmente se utiliza en esquemas de 1 dosis semanal en 3 de cada 4 sem y por tiempos prolongados ${ }^{23}$. Sus efectos secundarios más frecuentes son depresión de la médula ósea, anormalidades leves de la función hepática, síntomas similares a la influenza, exantema cutáneo, náuseas y vómitos ${ }^{3,4,14,19,21}$. La aparición de un SHU asociado al uso de GEM es un efecto adverso infrecuente, comunicándose sólo casos aislados o pequeñas series ${ }^{4-25}$. Las manifestaciones clínicas de esta complicación son la aparición de hipertensión arterial o el agravamiento de una existente, pudiendo presentarse como crisis hipertensiva o refractaria a tratamiento; anemia, púrpura, sangrado de mucosas, disnea, edema periférico, insuficiencia cardiaca, edema pulmonar no cardiogénico, derrame pleural, hematuria macroscópica, ictericia, exantema cutáneo, livedo reticularis, síntomas neurológicos (cefalea, confusión, visión borrosa) y fiebre $3-8,11,12,14,16,17,19,22$. Los exámenes de laboratorio característicamente

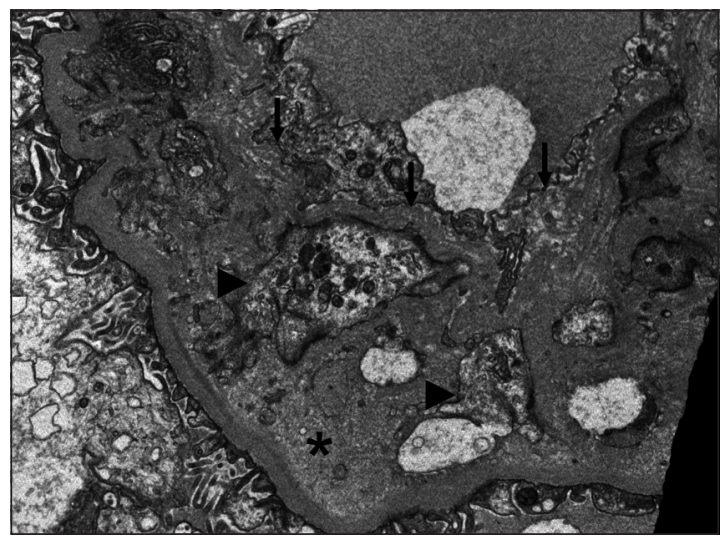

Figura 4. Microscopia electrónica de transmisión. Detalle de un doble contorno en un segmento de asa capilar libre. El endotelio está totalmente desprendido y alejado de la membrana basal original por expansión acentuada del espacio subendotelial debida a acumulación de material electron-lúcido en la lámina rara interna (asterisco), interposición citoplasmática celular (cabezas de flecha) y neoformación de material de membrana basal, el cual muestra un aspecto delaminado (flechas). (Tetróxido de osmio-acetato de uranilo, x8.000).

muestran anemia hemolítica microangiopática (esquistocitos, reticulocitos elevados, hiperbilirrubinemia de tipo indirecto, test de Coombs negativo, LDH elevada, haptoglobina baja o indetectable); trombocitopenia, ausencia de coagulación intravascular diseminada (fibrinógeno, TTPK y tiempo de protrombina normales); y disfunción renal (azotemia, proteinuria, hematuria, cilindruria hemática) que a menudo es severa y requiere

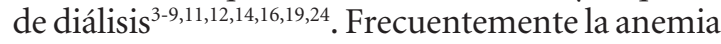
y la trombocitopenia se atribuyen erróneamente a la depresión medular originada por GEM advirtiéndose tardíamente la presencia de un $\mathrm{SHU}^{3}$. En este paciente la aparición aguda de un síndrome nefrítico hizo sospechar una nefropatía asociada al uso de GEM, motivando la realización de una biopsia renal. En él, las alteraciones hematológicas (anemia progresiva y trombocitopenia) habían comenzado a las 6 sem de iniciada la quimioterapia, lo que erróneamente se había atribuido a depresión de la médula ósea por GEM.

El SHU asociado a GEM usualmente se presenta después de 5-7 meses de su uso, posterior a la dosis $\mathrm{n}^{\circ} 18$ o al haber alcanzado una dosis acumulada superior a los $20.000 \mathrm{mg} / \mathrm{m}^{2} 3,5,11,12,14-$ $16,19,24$. Sin embargo, hay comunicaciones con 
dosis menores e incluso con una dosis única ${ }^{15}$. En este paciente las alteraciones hematológicas aparecieron después de la quinta dosis de GEM (1.600 mg por vez). La administración de GEM simultáneamente con otras drogas oncológicas y el uso previo de otros esquemas de quimioterapia predisponen a esta complicación ${ }^{10,11,19,20}$. Este paciente, que no había sido tratado previamente con ningún medicamento oncológico, había recibido una dosis acumulada de GEM de $16.400 \mathrm{mg}$ ( 9.318 $\mathrm{mg} / \mathrm{m}^{2}$ ) hasta la aparición del síndrome nefrítico.

La fisiopatología del SHU asociado a GEM no está bien aclarada existiendo varias hipótesis. Lo central es el daño de la célula endotelial ${ }^{12}$. Una de las hipótesis es de tipo inmune y plantea que existe formación de complejos inmunes circulantes, que gatillarían la agregación y depósito de plaquetas en áreas de daño endotelial en la microvasculatura renal o la formación de anticuerpos contra antígenos propios después de producirse un daño endotelial por la droga ${ }^{10}$. Otra hipótesis postula que la droga produce directamente una injuria a la célula endotelial de los capilares, generando edema celular, separación del endotelio de su membrana basal, neoformación de la membrana basal $\mathrm{y}$ activación de plaquetas y neutrófilos ${ }^{10,12}$. Otra hipótesis plantea que se producirían anticuerpos contra la enzima ADAMTS-13, lo que impediría fraccionar los grandes complejos del factor von Willebrand, activándose las plaquetas originando trombosis en los capilares ${ }^{8,10,12,17,22,24}$.

Cuando se efectúa una biopsia renal característicamente se encuentra una microangiopatía trombótica (MAT). Ella se caracteriza en la microscopia de luz por engrosamiento difuso y uniforme de la pared capilar por duplicación de las membranas basales (como huella de camión), edema de células endoteliales, trombos plaquetarios intraluminales, oclusión focal de capilares glomerulares y arteriolas por trombos de fibrina, expansión del mesangio y engrosamiento de arteriolas con proliferación miointimal ${ }^{3,4}$. La inmunofluorescencia muestra de manera variable depósitos de complemento C'3 e inmunoglobulinas en arterias pequeñas y arteriolas, sugiriendo una base inmune $^{26}$. La ultramicroscopia característicamente muestra en los capilares glomerulares, edema de la célula endotelial con desprendimiento de su membrana basal, depósito de material granular en el espacio subendotelial, a veces con fibrina en tactoides. Puede haber reticulación de la matriz mesangial y mesangiolisis. En las formas más agudas se observan trombos de fibrina en arterias, arteríolas y capilares glomerulares y un material mucoide en la íntima de arterias y arteriolas. En la forma crónica se observa remodelación de las paredes capilares y mesangio, dobles contornos que muestran interposición celular y expansión mesangial por acumulación de material de matriz a manera de cicatrización. Las arterias y arteriolas pueden mostrar esclerosis y una pared vascular en multicapas de miocélulas descrita como en "tela de cebolla". A menudo hay una sobreposición de elementos de una MAT aguda y crónica como sucedió en este caso (Figura 1 y 2 ).

En el tratamiento de esta afección se han empleado numerosas medidas con resultados variables $^{24}$. La principal medida terapéutica, en la que hay acuerdo unánime entre los autores, es la suspensión de la administración de GEM. En ocasiones esta sola medida revierte el trastorno, en otras, el SHU secundario no se detiene, dañándose irreversiblemente el riñón dejando al enfermo en diálisis crónica o provocando su muerte ${ }^{24}$. Entre los agentes empleados en la terapia destacan los hipotensores, diuréticos, corticoides, vincristina, azatioprina, plasmaféresis, recambios sanguíneos, transfusiones, plasma fresco congelado, prostaciclina, inmunoglobulinas, antiplaquetarios y rituximab $3,5,10-12,17,20,22$. Con esta última droga hay escasa experiencia pero los resultados parecen promisorios ${ }^{10}$. En este paciente dada la disfunción renal moderada se optó por discontinuar definitivamente la GEM y tratarlo sólo con hipotensores y diuréticos, para manejar la hipertensión arterial y el edema. Estas medidas permitieron la normalización de los parámetros hematológicos y una mejoría de los renales, continuando con la administración de hipotensores por la persistencia de hipertensión arterial.

La mortalidad comunicada en los pacientes con SHU asociado a GEM ha variado entre 15 y $70 \%{ }^{14,18,21}$. La interpretación de estas cifras es difícil, ya que muchos enfermos fallecieron como consecuencia del cáncer y no necesariamente del SHU.

El motivo de la comunicación de este caso clínico es advertir sobre la existencia de este grave y poco conocido efecto secundario de la GEM y proponer algunas medidas para su diagnóstico oportuno. Nos parece prudente medir la presión arterial y contar con determinaciones de crea- 
tinina, bilirrubina total y directa, hemoglobina, recuento plaquetario, recuento de reticulocitos, $\mathrm{LDH}$, haptoglobina, un frotis sanguíneo (buscando esquistocitos) y un sedimento urinario; antes de administrar una nueva dosis de GEM. Estas medidas deben emplearse especialmente en los pacientes que reciben GEM por más de 3 meses, reciben cisplatino conjuntamente, tienen anemia y/o trombocitopenia, desarrollan edema, congestión pulmonar, hematuria o hipertensión $\operatorname{arterial}^{3,17}$.

\section{Referencias}

1. Noris M, Remuzzi G. Hemolytic uremic syndrome. J Am Soc Nephrol 2005; 16: 1035-50.

2. Copelovitch L, Kaplan BS. The thrombotic microangiopathies. Pediatr Nephrol 2008; 23: 1761-7.

3. Humphreys BD, Sharman JP, Henderson JM, Clark JW, Marks PW, Rennke HG, et al. Gemcitabine-associated thrombotic microangiopathy. Cancer 2004; 100: 266470 .

4. Flombaum CD, Mouradian JA, Casper ES, Erlandson RA, Benedetti F. Thrombotic microangiopathy as a complication of long-term therapy with gemcitabine. Am J Kidney Dis 1999; 33: 555-62.

5. Brodowicz T, Breiteneder S, Wiltschke C, Zielinski CC. Gemcitabine-induced hemolytic uremic syndrome: a case report. J Natl Cancer Inst 1997; 89: 1895-6.

6. Choi M, Woywodt A, Göbel U, Schneider W, Kettritz R. Haemolytic uraemic syndrome after gemcitabine treatment for pancreatic carcinoma. Nephrol Dial Transplant 1999; 14: 2523-4.

7. Phelan PJ, Liew A, Magee C. A case of progressive hypertension preceding gemcitabine-associated thrombotic microangiopathy complicated by acute kidney injury and stroke. Ren Fail 2009; 31: 743-4.

8. Thomas JG, Sethi S, Norby SM. Chronic thrombotic microangiopathy secondary to chemotherapy for urothelial carcinoma in a patient with a history of Wegener granulomatosis. Am J Kidney Dis 2011; 57: 799-802.

9. Nackaerts K, Daenen M, Vansteenkiste J, Vandevelde A, Van Bleyenbergh P, Demedts M. Hemolytic-uremic syndrome caused by gemcitabine. Ann Oncol 1998; 9: 1355-6.

10. Gourley BL, Mesa H, Gupta P. Rapid and complete resolution of chemotherapy-induced thrombotic thrombocytopenic purpura/hemolytic uremic syndrome (TTP/ HUS) with rituximab. Cancer Chemother Pharmacol 2010; 65: 1001-4.
11. Glezerman I, Kris MG, Miller V, Seshan S, Flombaum $\mathrm{CD}$. Gemcitabine nephrotoxicity and hemolytic uremic syndrome: report of 29 cases from a single institution. Clin Nephrol 2009; 71: 130-9.

12. Izzedine $\mathrm{H}$, Isnard-Bagnis $\mathrm{C}$, Launay-Vacher $\mathrm{V}$, Mercadal L, Tostivint I, Rixe O, et al. Gemcitabine-induced thrombotic microangiopathy: a systematic review. Nephrol Dial Transplant 2006; 21: 3038-43.

13. Arnadottir M, Benediktsson T, Hrafnkelsson J. The cumulative incidence of gemcitabine-induced thrombotic microangiopathy. Acta Oncológica 2007; 46: 545-6.

14. Willemsen AECA, van Herpen CML, Wesseling P, Bult $\mathrm{P}$, van Laarhoven HWM. Fatal thrombotic microangiopathy after a single dose of gemcitabine as fourth-line palliative treatment for metastasized ductal breast carcinoma. Acta Oncológica 2011; 50: 462-5.

15. De Smet D, Jochmans K, Neyns B. Development of thrombotic thrombocytopenic purpura after a single dose of gemcitabine. Ann Hematol 2008; 87: 495-6.

16. Lhotta K, Kühr T, Rumpelt HJ, Wöll E, Thaler J, König P. Thrombotic microangiopathy with renal failure in two patients undergoing gemcitabine chemotherapy. Am J Nephrol 1999; 19: 590-3.

17. Bharthuar A, Egloff L, Becker J, George M, Lohr JW, Deeb G, et al. Rituximab-based therapy for gemcitabineinduced hemolytic uremic syndrome in a patient with metastatic pancreatic adenocarcinoma: a case report. Cancer Chemother Pharmacol 2009; 64: 177-81.

18. Ruiz I, Del Valle J, Gómez A. Gemcitabine and haemolytic uraemic syndrome. Ann Oncol 2004; 15: 1575-6.

19. Zupancic M, Shah PC, Shah-Khan F. Gemcitabineassociated thrombotic thrombocytopenic purpura. Lancet Oncol 2007; 8: 634-41.

20. Müller S, Schütt P, Bojko P, Nowrousian MR, Hense J, Seeber S, et al. Hemolytic uremic syndrome following prolonged gemcitabine therapy: report of four cases from a single institution. Ann Hematol 2005; 84: 6212.

21. Walter RB, Joerger M, Pestalozzi BC. Gemcitabineassociated hemolytic-uremic syndrome. Am J Kidney Dis 2002; 40: E 16.

22. Fung MC, Storniolo AM, Nguyen B, Arning M, Brookfield W, Vigil J. A review of hemolytic uremic syndrome in patients treated with gemcitabine therapy. Cancer 1999; 85: 2023-32.

23. Casper ES, Green MR, Kelsen DP, Heelan RT, Brown TD, Flombaum CD, et al. Phase II trial of gemcitabine (2,2'-difluorodeoxycystidine) in patient with adenocarcinoma of the pancreas. Invest New Drugs 1994; 12 : 29-34.

24. Gore EM, Jones BS, Marques MB. Is therapeutic plasma 
exchange indicated for patients with gemcitabineinduced hemolytic uremic syndrome? J Clin Apheresis 2009; 24: 209-14.

25. Erke S, Riess H, Oettle H, Huhn D. Elevated reticulocyte count - a clue to the diagnosis of haemolytic-uraemic syndrome (HUS) associated with gemcitabine therapy for metastatic duodenal papillary carcinoma: a case report. Br J Cancer 1999; 79: 1519-21.

26. Onitilo AA, Engel JM, Clouse LH, Gerndt KM. Successful treatment of mitomycin-induced thrombotic thrombocytopenic purpura with rituximab. J Vasc Interv Radiol 2009; 20: 275-6. 